\title{
Validation of Hadronic Models in Geant4
}

\author{
Tatsumi Koi, Dennis H. Wright ·, Gunter Folger, Vladimir Ivantchenko, \\ Mikhail Kossov, Nikolai Starkov†, Aatos Heikkinen * , Pete Truscott , \\ Fan Lei††, and Hans-Peter Wellischł \\ -Stanford Linear Accelerator Center, Menlo Park, California, USA \\ †CERN, Geneva, Switzerland \\ - Helsinki Institute of Physics, Helsinki, Finland \\ †+QinetiQ, Farnborough, UK \\ $\ddagger$ Geneva, Switzerland
}

\begin{abstract}
Geant 4 is a software toolkit for the simulation of the passage of particles through matter. It has abundant hadronic models from thermal neutron interactions to ultra relativistic hadrons. An overview of validations in Geant4 hadronic physics is presented based on thintarget measurements. In most cases, good agreement is available between Monte Carlo prediction and experimental data; however, several problems have been detected which require some improvement in the models.
\end{abstract}

Keywords: simulation, Geant4

PACS: 24.10.Lx, 02.70.Uu, 01.30.Cc

\section{Introduction}

Abundant hadronic processes are available in Geant4 from thermal energy neutron interactions to energetic particle interactions available only at large accelerators or in cosmic rays. In some cases, there are multiple models for a given interaction so that users can select among them according to their requirements, in terms of application, precision and computing time. To aid user selection, validations of Geant 4 hadronic models will be shown in this paper and detailed explanations about several selected models are available in another presentation [1] at this conference. Thin target experimental data are mainly used, because they allow a clean and detailed study of single hadronic interactions. Another type of validation uses calorimeter test-beam data with the complete detector setup. In this case, the observables are the convolution of many interactions; therefore, whole functions of Geant4 are validated at once. However, for the validation of each hadronic model, comparison to thin-target measurements is more suitable. The validations of Geant4 against calorimeter testbeam data were presented by other authors at this conference.

The coverage of Geant4 hadronic physics is quite wide and the number of hadronic models is also large. Due to space limitations however, not all models are represented and only one or a few validation plots are presented for each one. In the following sections, we will show validation results of Geant4 hadronic physics beginning with low energy models that include capture and pre-compound models and proceeding to high energy. Then we will treat special topics such as gamma-nuclear, low energy neutron and ion-ion interactions. 


\section{Low energy interactions}

In this section, "low energy" refers to models in which the reaction energy is lower than the validity range of the Intra- Nuclear Cascade models. In addition to hadron scattering from a target nucleus, capture on the nucleus is also treated. The Chiral Invariant Phase Space (CHIPS) model [2] shows the best agreement to capture data. Fig. 1 shows comparisons of CHIPS model results with the experimental spectra [3, 4] of secondary particles and nuclear fragments from negative pion capture on ${ }^{12} \mathrm{C}$ nucleus. They are plotted as a function of $k=\left(p+E_{k i n}\right) / 2$, where $E_{\text {kin }}$ and $p$ are the kinetic energy and the momentum of the secondary particles. Good agreement is observed in all energy ranges for all types of secondary particles. Other models are also available for this interaction, however they are not as detailed.

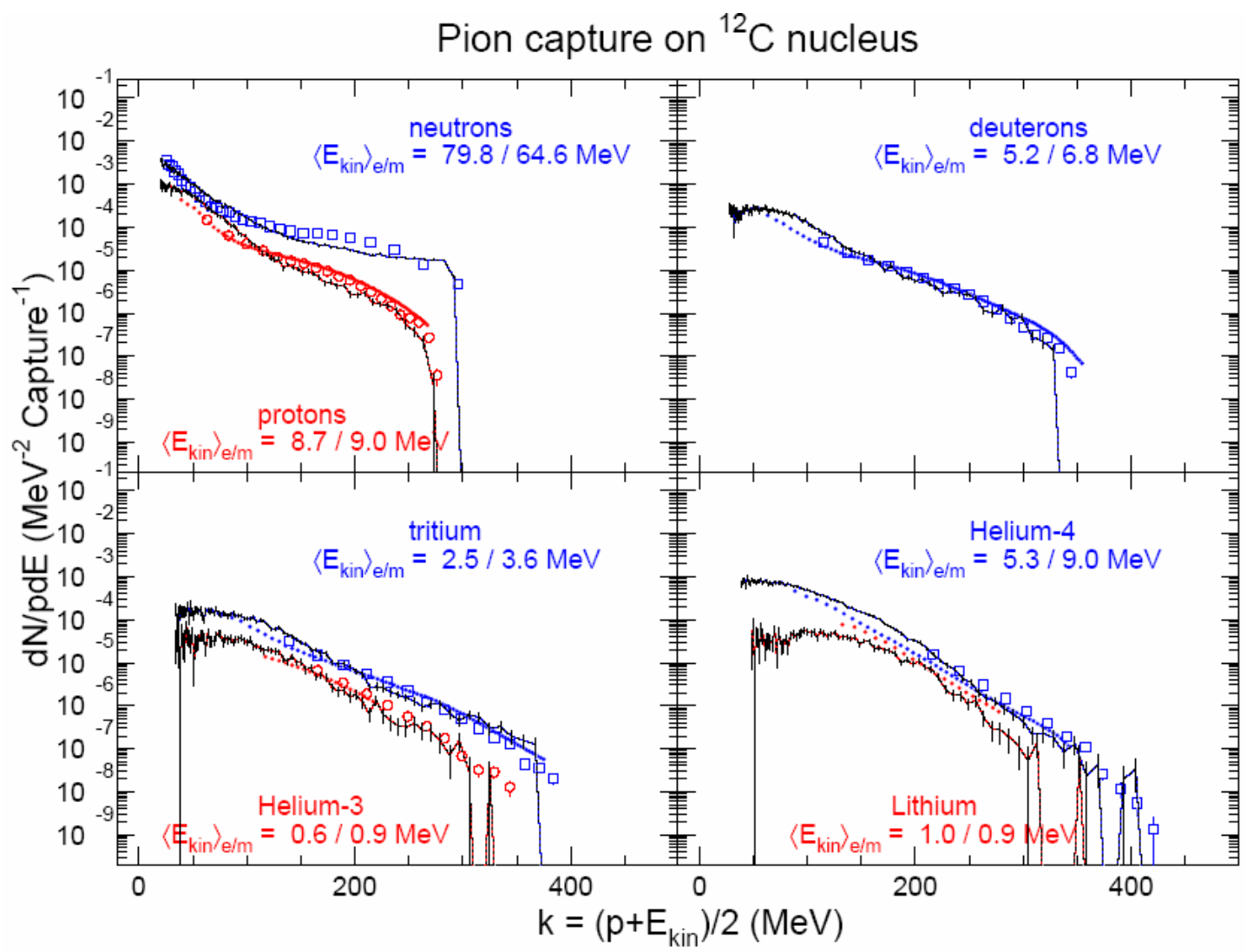

FIGURE 1. Comparison of the CHIPS model results with experimental data on proton, neutron, and nuclear fragment production in the capture of negative pions on $\mathrm{C}$. The experimental neutron spectrum is taken from papers [3, 4]. The model calculations are shown by the two corresponding solid lines. The average kinetic energy carried away by each nuclear fragment is shown in the panels by the two numbers: first is the average calculated using the experimental data shown; second is the model result.

The pre-compound model, which can be divided into an exciton part and an evaporation part, is often used for hadron-nucleus interactions in this energy region.

Several pre-compound models are available in Geant4. Most of them are currently integrated within specific models such as the Beritin-style cascade [5], CHIPS model and Low Energy Parameterization (LEP) model which is based on the GHEISHA model of Geant3. There is also an independently implemented pre-compound model, 
which may be used by itself or coupled to Binary cascade [6] and/or high energy models of Geant4. Validations of the independently implemented pre-compound model are given in Fig. 2. The plots show neutron production cross sections resulting from protons bombarding tin and bismuth targets. Secondary neutrons created in the exciton and evaporation parts are presented separately in the plots. There is good agreement for both reactions.

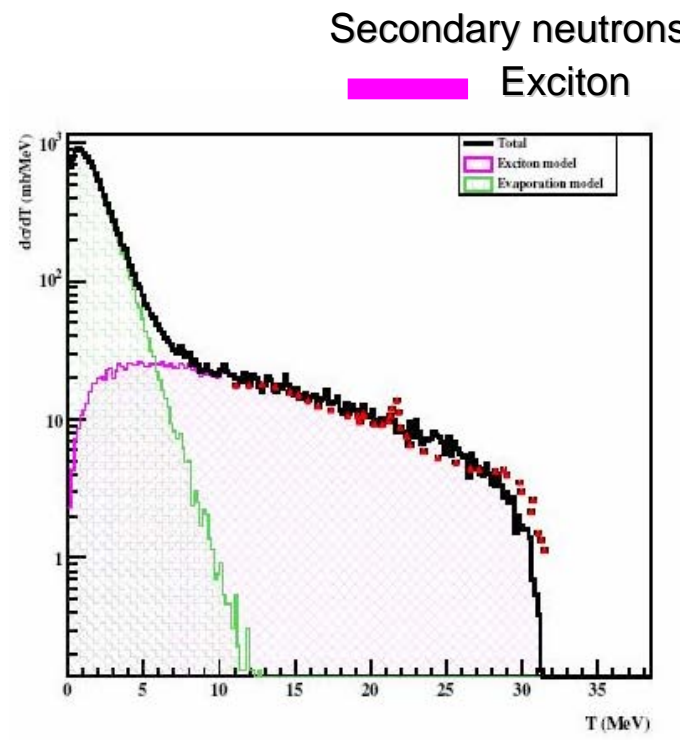

$\mathrm{Sn}(\mathrm{p}, \mathrm{X}$ n) 35MeV

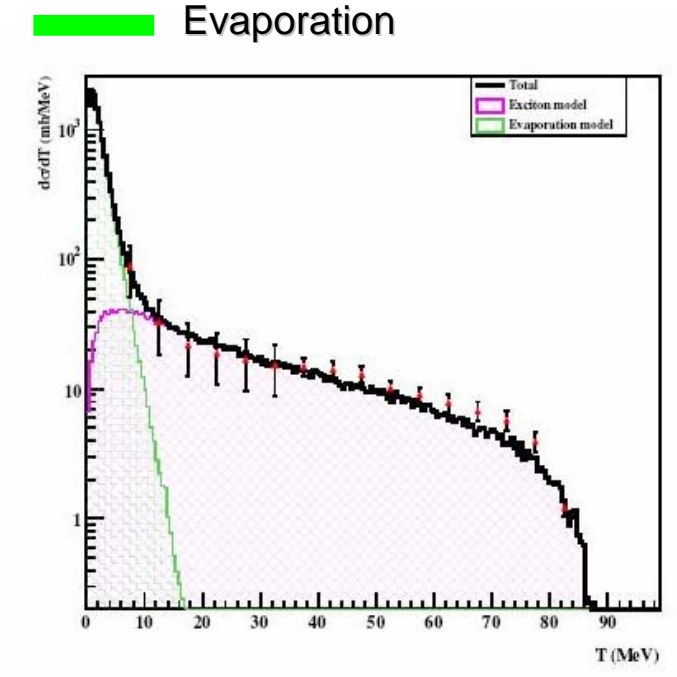

$\mathrm{Bi}(\mathrm{p}, \mathrm{X} n) 90 \mathrm{MeV}$

FIGURE 2. Comparisons of inclusive neutron production cross sections resulting from protons bombarding Sn and Bi targets [7]. The predicted flux is divided into neutrons generated by the exciton model (pink line) and the evaporation model (green line). 
Cross section(mb) ws A (for Element Z) in $\mathrm{Al}(\mathrm{p}, \mathrm{X})$ of $800 \mathrm{MeV}$
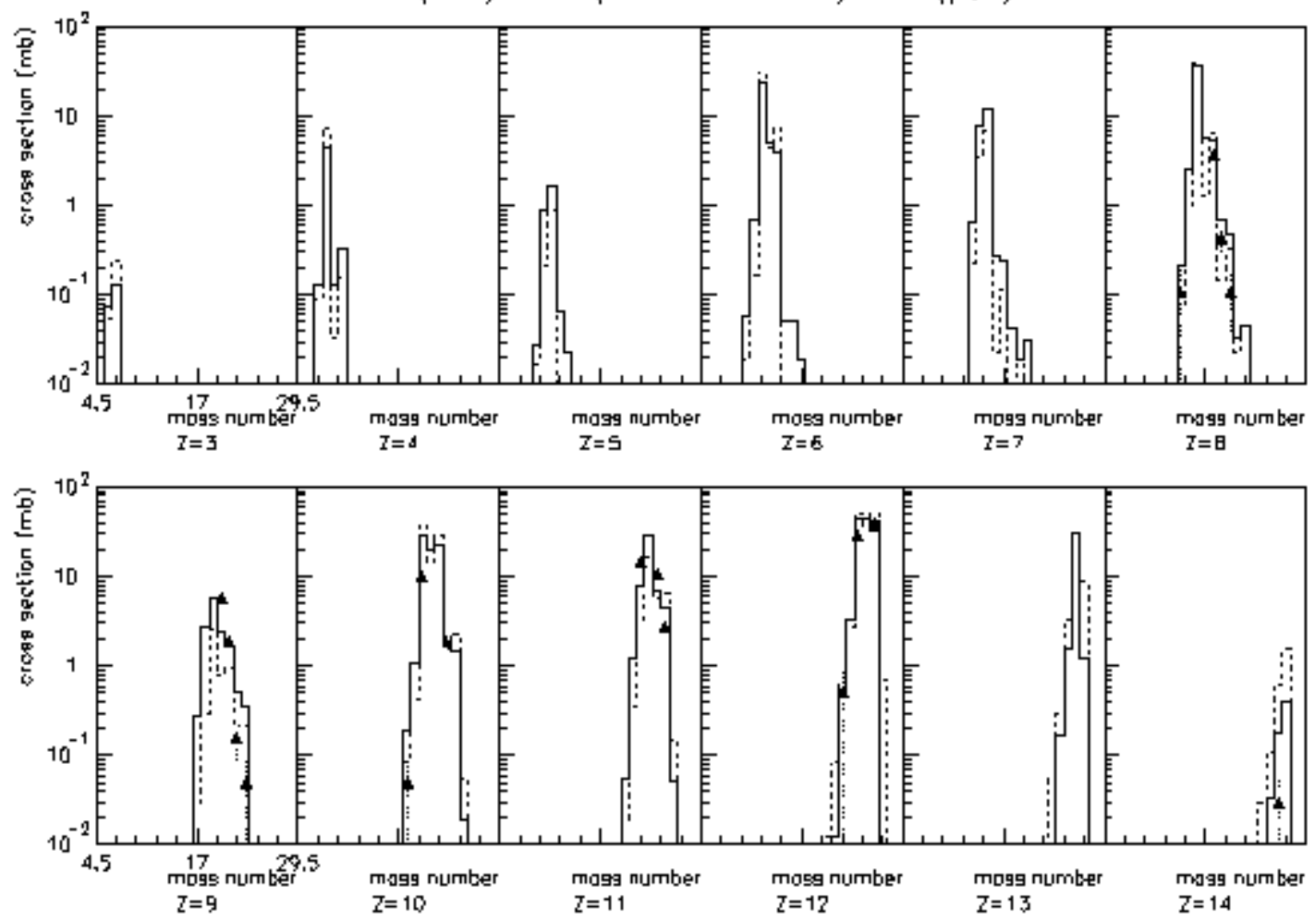

FIGURE 3. Comparison of isotope production resulting from $800 \mathrm{MeV}$ proton bombarding aluminum among Geant4 models and data [8]. Histogram - prediction of Bertini cascade (solid line) and Binary cascade (dashed line). Triangle -experimental data. Intermediate energy interactions

Isotope production by nuclear interactions is also treated in this section, because the pre-compound model usually plays the most important role in the isotope distribution from highly excited nuclei. Fig. 3 shows the validation of isotope production resulting from protons bombarding aluminum. Monte Carlo predictions are obtained from the Bertini and Binary cascade models. As already mentioned, different pre-compound models are used within each of these cascade models. The plot shows that the two precompound models work equally well.

\section{Intermediate energy interactions}

Intra-Nuclear Cascade models are generally used for simulation of hadron interactions above a few tens of $\mathrm{MeV}$. The upper limits for these are model dependent. As mentioned before, Geant4 has two cascade models, namely a "Bertini-like cascade" and a "Binary cascade". The upper limit of the former model is roughly 10 $\mathrm{GeV}$ and the later is about $3 \mathrm{GeV}$. LEP models are also available for this energy region. A verification suite has been created in this energy region [9] which includes a large set of experimental data from thin-target scattering experiments. Fig. 4 is an example of a plot obtained using the suite. It shows the validation of Binary and Bertini cascade models for double differential neutron production cross sections. In most cases good agreement is found, however relatively large disagreements exist at 
the most forward scattering angles in the Bertini cascade. In the Binary cascade, agreement at forward angles is better than at backward angles.

Binary Cascade
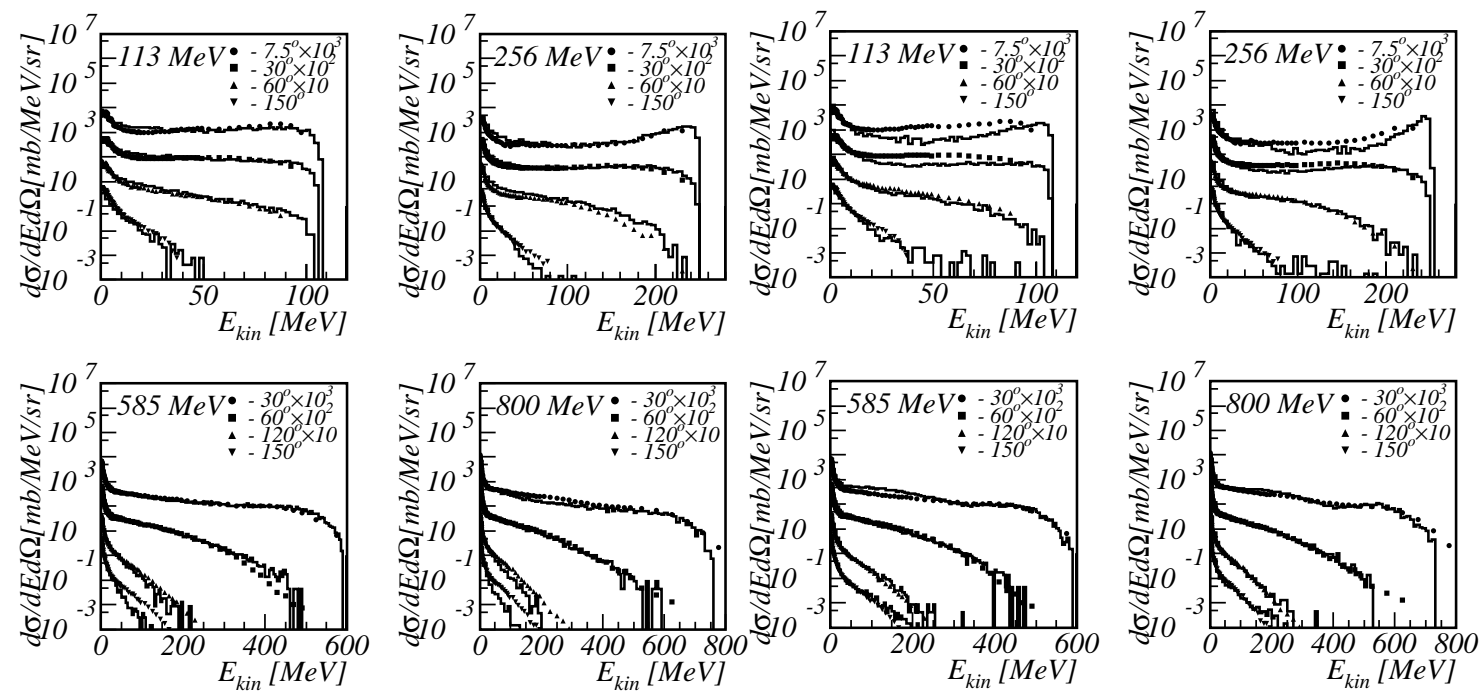

FIGURE 4. Double differential cross-section for neutrons produced in proton scattering off aluminum. Histograms -Monte Carlo predictions. Points-Data [10].

\section{High energy interactions}

The Geant4 has two parton string models, namely the Quark-Gluon String (QGS) and Fritiof (FTF). These models are able to calculate hadronic interactions with reaction energies above a total center-of-mass energy of $5 \mathrm{GeV}$. Between the upper limit of our cascade models and the lower limit of these models, currently Geant 4 has only the LEP model. The High Energy Parameterized (HEP) model (also based on GHEISHA) is available for hadronic interactions above $20 \mathrm{GeV}$. Fig. 5 is a validation plot of transverse momentum (Pt) and rapidity distributions of secondary pions resulting from pion-Magnesium reactions at a laboratory momentum of $320 \mathrm{GeV} / \mathrm{c}$. In the Pt distribution, the QGS model underestimates the data around the high Pt region. The rapidity distribution of the HEP model probably overestimates the peak and has an unphysical dip around 2. The peak position of QGS model is shifted slightly to the lower side. 

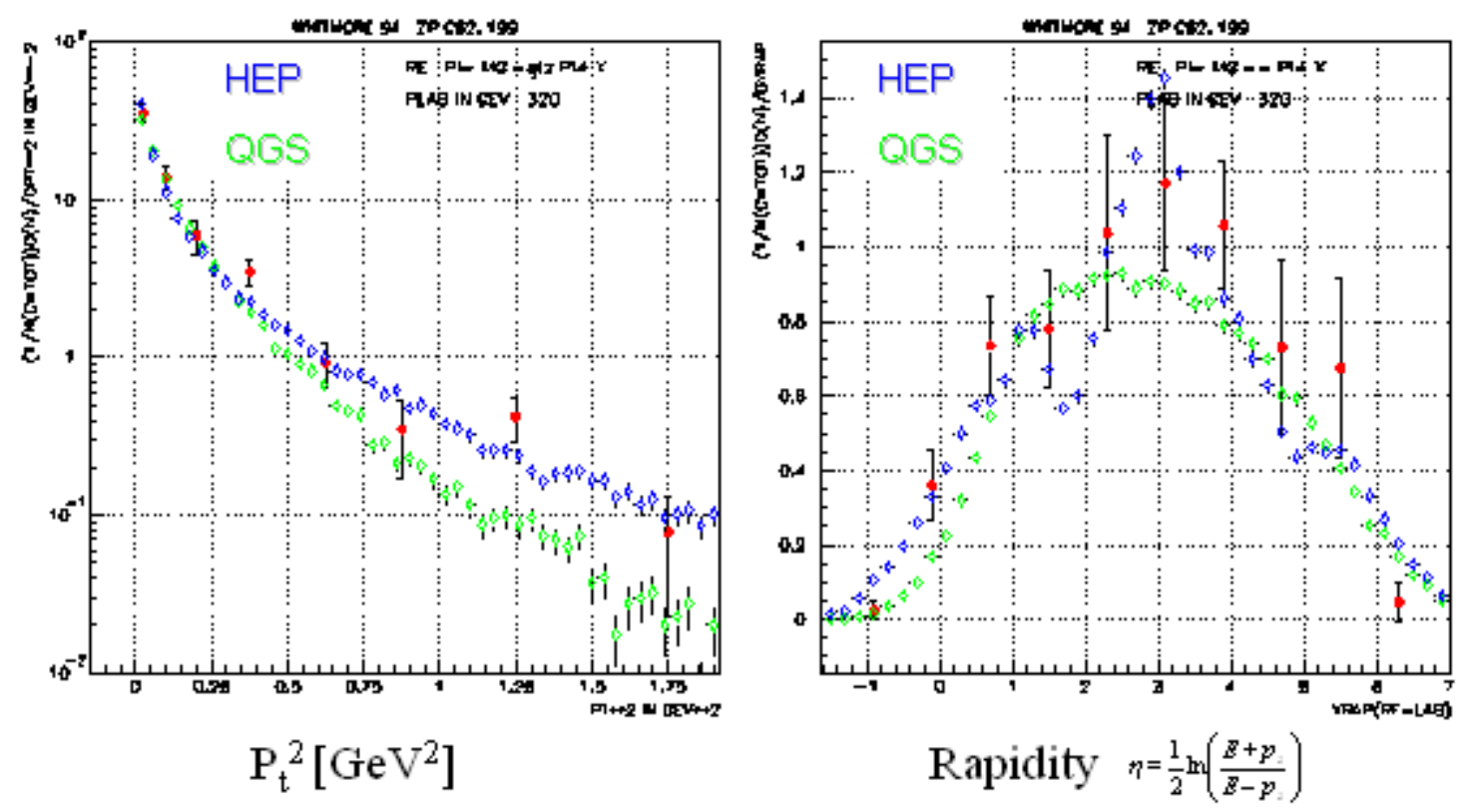

FIGURE 5. Comparison of Pt and rapidity distributions of secondary pions resulting from pionMagnesium reactions at laboratory momentum of $320 \mathrm{GeV} / \mathrm{c}$. Open blue diamonds - prediction of HEP model. Open green diamonds - predictions of QGS model. Red box - experimental data[11].

\section{Photon-nucleAr interactions}

The CHIPS model in Geant4 can also apply to the calculation of complicated mechanisms of interaction between photons and hardons in nuclear matter. Fig. 6 shows comparison of the CHIPS model results with the experimental data [12, 13]. Proton yields from about $60 \mathrm{MeV}$ gamma-rays bombarding ${ }^{40} \mathrm{Ca}$ are shown in the figure. The invariant inclusive cross sections $\left(d \sigma / p_{p} d E_{p} d \Omega_{p}\right)$ of secondary protons are plotted as a function of $k=\left(p+E_{k i n}\right) / 2$, where $E_{k i n}$ and $p$ are the kinetic energy and the momentum of the secondary protons. The angular dependence of the proton yield in photoproduction is reproduced quite well. The Low Energy Parameterized (LEP) model is also available for this interaction, however it is not as detailed. 


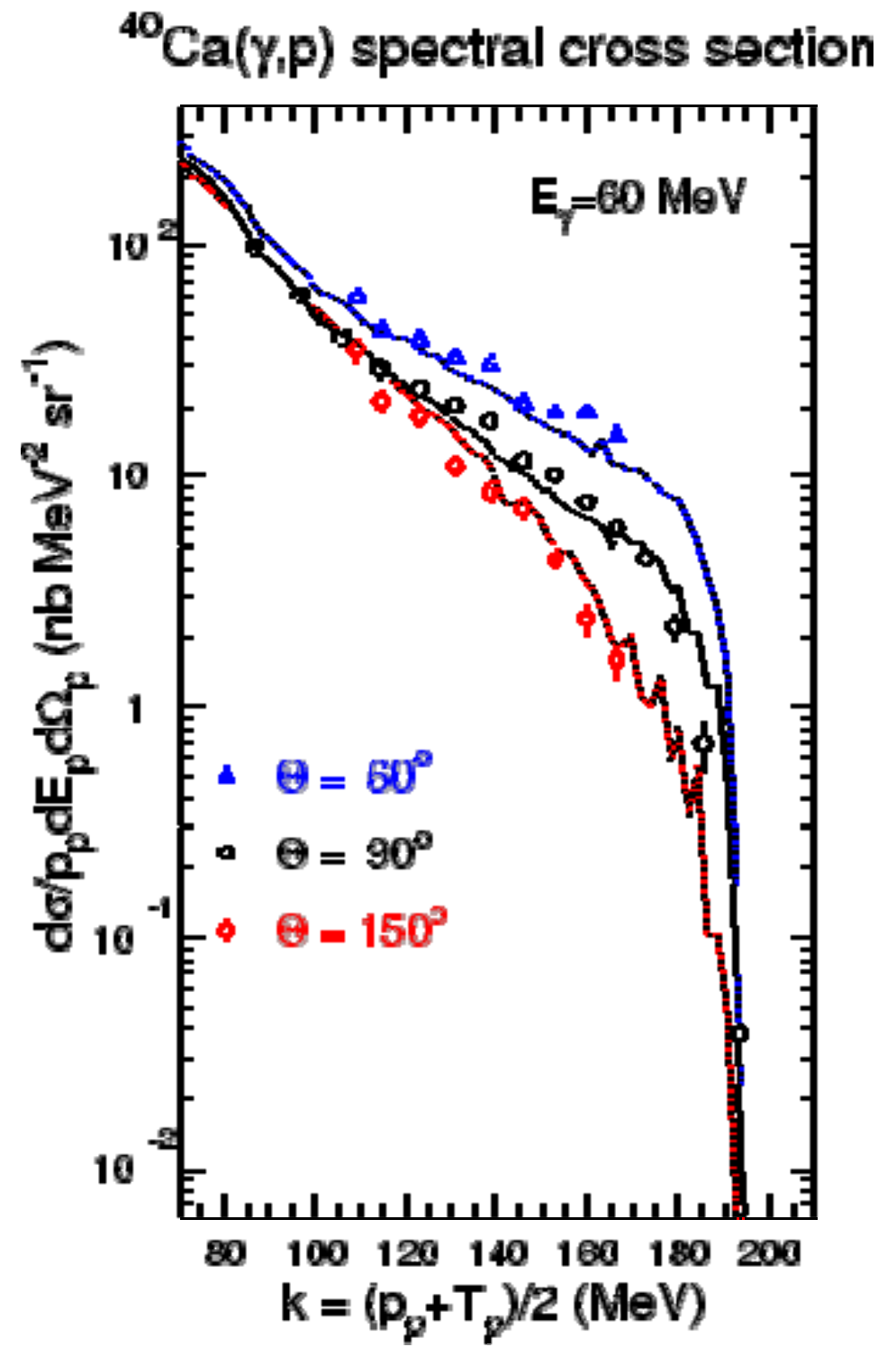

FIGURE 6. Comparison of CHIPS model with experimental data on proton production in photonuclear reactions on $\mathrm{Ca}$ at $59-65 \mathrm{MeV}$. Open circles, triangles and diamonds represent the experimental proton spectra. Lines show the results of the corresponding CHIPS model calculation. 


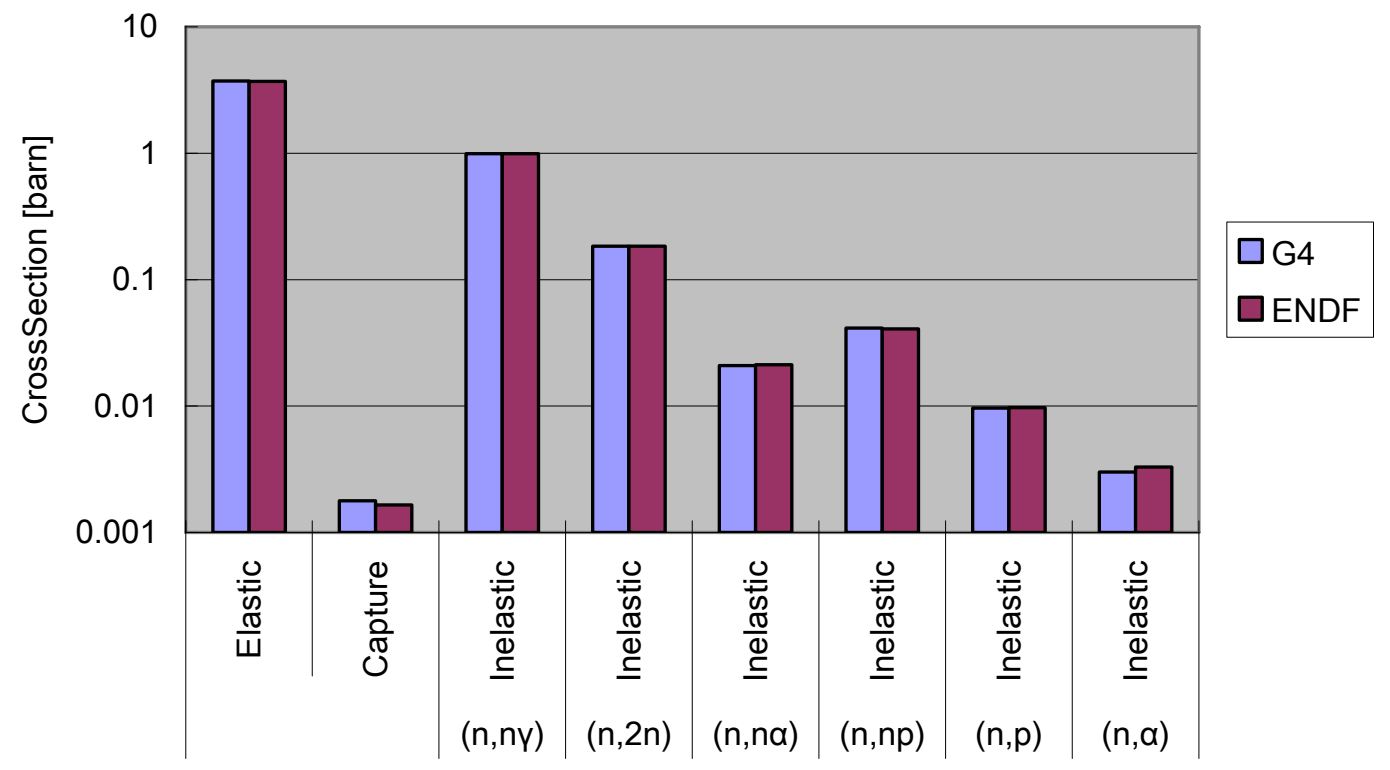

FIGURE 7. Comparison of channel cross sections between simulation results and reference data of ENDF/B-VI release8. Simulation results derived from thin-target calculation with Neutron HP models.

\section{Ion interactions}

Geant4 has several models for nucleus-nucleus interactions. The Binary Light Ion Cascade model is an extension of the Binary cascade model. It can handle ion interactions up to $3 \mathrm{GeV} / \mathrm{n}$ when the mass number of the projectile and/or target nucleus is less than 12. This limitation in mass number of the reaction system is not strict and in some cases the model can predict the final state well beyond the limitation [16]. Fig. 8 shows the neutron yield from $400 \mathrm{MeV} / \mathrm{n}{ }^{56} \mathrm{Fe}$ beams bombarding thick carbon and aluminum targets. Reactions between ${ }^{56} \mathrm{Fe}$ and $\mathrm{Al}$ nuclei are beyond the limitation while reactions between ${ }^{56} \mathrm{Fe}$ and $\mathrm{C}$ nuclei are within the limitation. However, judging from the validation plots, no significant difference in agreement can be seen between the two reactions.

The Abrasion-Ablation model is a C++ implementation of NUCFRG2 [17] physics and is also available in Geant4. This is a simplified macroscopic model for nucleusnucleus interactions up to $10 \mathrm{GeV} / \mathrm{n}$.

There is another kind of nucleus-nucleus interaction, namely electromagnetic dissociation. It is the liberation of nucleons or nuclear fragments as a result of the electromagnetic field by exchange of virtual photons. The EM Dissociation model of Geant 4 can handle this interaction. Table 1 shows a validation of the EM Dissociation model for ultra relativistic ion projectiles in nuclear emulsion. Monte Carlo predictions are within experimental error bars in most cases. 

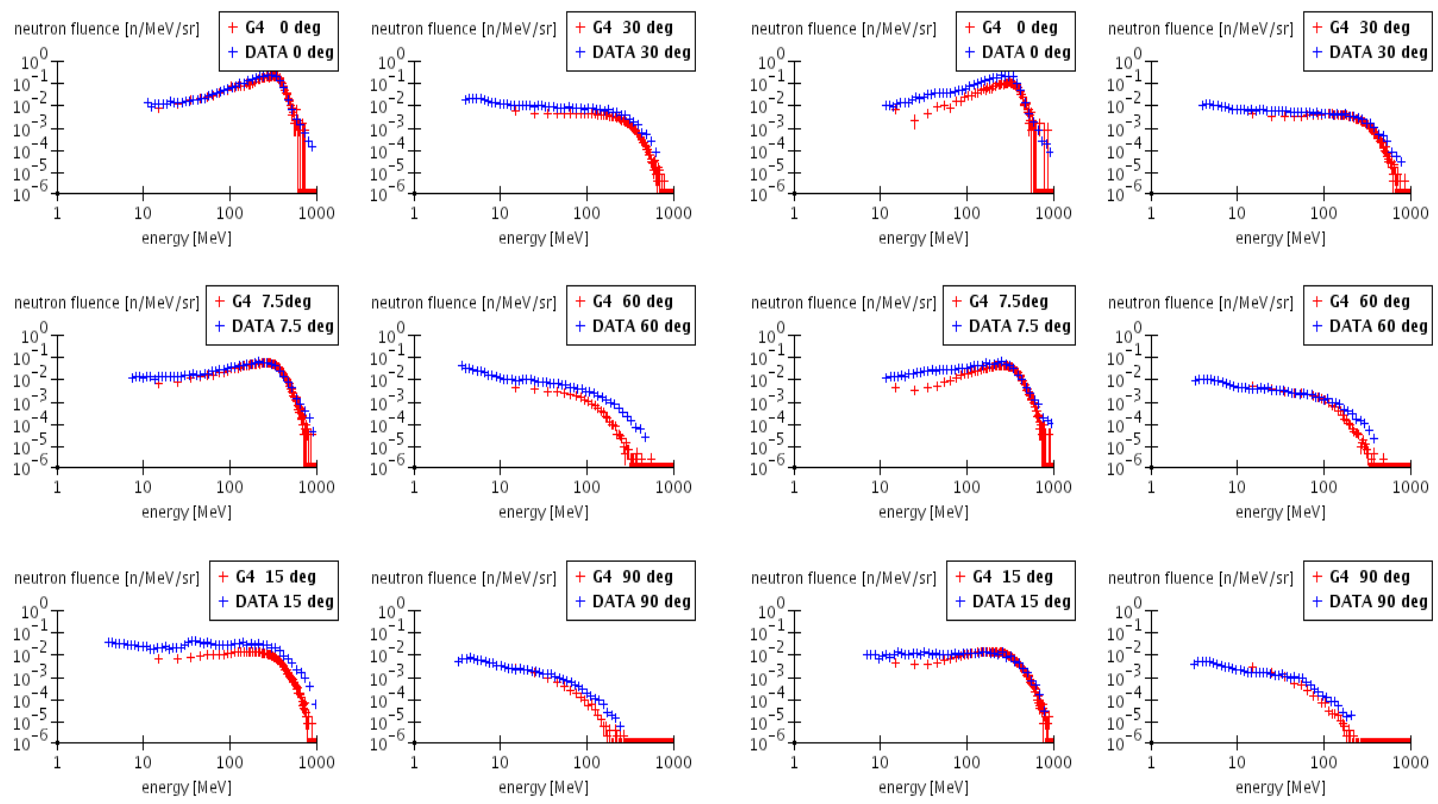

FIGURE 8. Comparison between data [18] and Geant4 simulation of double differential neutron production for a Fe beam of $400 \mathrm{MeV} / \mathrm{n}$ on thick Carbon and Aluminum targets.

TABLE 1. Comparison of cross-sections for ED interactions in silver in which projectile produces a single proton Experimental data are as reported by Jilany [19]

\begin{tabular}{lcccc} 
Projectile & $\begin{array}{c}\text { Energy } \\
\text { [GeV/nuc] }\end{array}$ & $\begin{array}{c}\text { Product from } \\
\text { ED }\end{array}$ & $\begin{array}{c}\text { G4EM } \\
\text { Dissociation } \\
\text { [mbarn] }\end{array}$ & $\begin{array}{c}\text { Experiment } \\
\text { [mbarn] }\end{array}$ \\
\hline Mg-24 & 3.7 & $\mathrm{Na}-23+\mathrm{p}$ & $124 \pm 2$ & $154 \pm 31$ \\
Si-28 & 3.7 & $\mathrm{Al}-27+\mathrm{p}$ & $107 \pm 1$ & $186 \pm 56$ \\
& 14.5 & $\mathrm{Al}-27+\mathrm{p}$ & $216 \pm 2$ & $165 \pm 24$ \\
$\mathrm{O}-16$ & $\mathrm{~N}-15+\mathrm{p}$ & $331 \pm 2$ & $128 \pm 33$ \\
& & & & $342 \pm 22$ \\
\hline
\end{tabular}

\section{Conclusion}

We have shown validations of low energy neutron, pre-compound, cascade, and high energy models. These are the most important for hadronic shower shapes and their agreement with data is good for most cases. However, relatively large disagreements are shown in the Pt distribution of the QGS model and the secondary neutron distribution at forward angles in the Bertini-like cascade model. These indicate that improvements in the diffractive part of the QGS model, and in the nuclear model of the Bertini-like cascade are required. The CHIPS model in Geant4 shows good agreement with data both for hadron capture and photonuclear interactions. The Binary Light Ion Cascade model has good agreement even for heavy ion collisions which are beyond its limitation in mass number, but improvement is needed in the correlation of participant nucleons and the transition to the pre-compound model. An 
extensive validation suite was prepared for the cascade energy region. Similar validation suites for other energy regions are required.

\section{Acknowledgments}

This work was supported by the Stanford Linear Accelerator Center (SLAC) through a grant from the U.S. Department of Energy. It was also supported by the European Organization for Nuclear Research (CERN) and the Helsinki Institute of Physics.

\section{References}

1. D. H. Wright, T. Koi, G. Folger, V. Ivanchenko, M. Kossov. N. Starkov. A. Heikkinen and H. P. Wellisch. in these proceedings of Hadronic Shower Simulation Workshop, Batavia, Illinois, 2006

2. M.V. Kossov, "Manual for the CHIPS event generator", KEK internal report 2000-17, Feb. 2001 H/R; P.V. Degtyarenko, M.V. Kossov and H.P. Wellisch, Eur. Phys. J. A8, 217 (2000); P.V. Degtyarenko, M.V. Kossov and H.P. Wellisch, Eur. Phys. J. A9 (2001); P.V. Degtyarenko, M.V. Kossov and H.P. Wellisch, Eur. Phys. J. A9 (2001).

3. G. Mechtersheimer et al., Nucl. Phys. A324, 379 (1979)

4. R. Madey, T. Villaithong, B. D. Anderson, J. N. Knudson, T. R. Witten, A. R. Baldwin, and F. M. Waterman, Phys. Rev. C25, 3050 (1982)

5. A.Heikkinen, N.Stepanov and HP.Wellisch, In the Proceedings of 2003 Conference for Computing in HighEnergy and Nuclear Physics (CHEP 03), La Jolla, California, 24-28 Mar 2003, pp MOMT008

6. G. Folger, V.N. Ivanchenko and J.P. Wellisch, Eur. Phys. J. A21, pp. 407, (2004)

7. A. M. Kalend, B. D. Anderson, A. R. Baldwin, R. Madey, J. W. Watson, C. C. Chang, H. D. Holmgren, R. W. Koontz, J. R. Wu, and H. Machner, Phys. Rev. C 28, 105 (1983)

8. H. Vonach, A. Pavlik, A. Wallner, M. Drosg, R. C. Haight, D. M. Drake, and S. Chiba, Phys. Rev. C55, 2458 (1997)

9. V. Ivanchenko, G.Folger, J. P. Wellisch, T. Koi and D. H. Wright In the Proceedings of 2003 Conference for Computing in High-Energy and Nuclear Physics (CHEP 03), La Jolla, California, 24-28 Mar 2003, pp MOMT009

10. M. M. Meier et al., "Differential neutron production cross sections and neutron yields from stopping-length targets for 113-MeV protons” Nucl. Scien. Engin. 102, 310, (1989); M. M. Meier et al., "Differential neutron production cross sections for 256-MeV protons" Nucl. Scien. Engin. 110, 289, (1992); W. B. Amian et al., "Differential neutron production cross sections for 597-MeV protons” Nucl. Scien. Engin. 115, 1, (1993) W. B. Amian et al., "Differential neutron production cross sections for 800-MeV protons” Nucl. Scien. Engin. 112, 78, (1992)

11. J. J. Whitmore, F. Persi, W. S. Toothacker, P. A. Elcombe, J. C. Hill, W. W. Neale, W. D. Walker, W. Kowald, P. Lucas, L. Voyvodic, R. Ammar, D. Coppage, R. Davis, J. Gress, S. Kanekal, N. Kwak, J. M. Bishop, N. N. Biswas, N. M. Cason, V. P. Kenney, M. C. K. Mattingly, R. C. Ruchti and W. D. Shephard, Z. Phys. C62 199-227 (1994)

12. D. Ryckbosh, L. van Horrebeke, R. Van de Vyver, F. De Smet, J. O. Adler, D. Nilsson, B. Schröder and R. Zorro, Phys. Rev. C42, 444 (1990)

13. P.D. Harty et al. (unpublished); (private communication). Cited in the reference: J. Ryckebusch, L. Machenil, M. Vanderhaeghen, V. Van der Sluys and M. Waroquier, Phys. Rev. C49, 2704 (1994).

14. J.P. Wellisch. “THE NEUTRON_HP NEUTRON TRANSPORT CODE” from Monte Carlo 2005 Topical Meeting ISBN: 0-89448-695-0 Chattanooga, Tennessee, April 17-21, 2005

15. ENDF/B-VI: Cross Section Evaluation Working Group, ENDF/B-VI Summary Document, Report BNL-NCS-17541 (ENDF-201) (1991), edited by P.F. Rose, National Nuclear Data Center, Brookhaven National Laboratory, Upton, NY, USA.

16 T. Koi et al., “ION TRANSPORT SIMULATION USING GEANT4 HADRONIC PHYSICS” from Monte Carlo 2005 Topical Meeting ISBN:0-89448-695-0 Chattanooga, Tennessee, April 17-21, 2005

17. J. W. Wilson, R. K. Tripathi, F. A. Cucinotta, J. L. Shinn, F. F. Badavi, S. Y. Chun, J. W. Norbury, C. J. Zeitlin, L. Heilbronn, and J. Miller, "NUCFRG2: An Evaluation of the Semiempirical Nuclear Fragmentation Database.” NASA TP-3533, 1995.

18 T. Kurosawa, N. Nakao, T. Nakamura, H. Iwase, H. Sato, Y. Uwamino, and A. Fukumura, Phys. Rev. C62, 044615 (2000). 
19. M.A. Jilany; Nuclear Physics A705 477-493 (2002). 